Check for updates

Cite this: RSC Adv., 2018, 8, 6931

\section{Stability and electronic structure of hydrogen vacancies in ADP: hybrid DFT with vdW correction}

\author{
Tingting Sui, Yafei Lian, Mingxia Xu, Lisong Zhang, Yanlu Li, (DD * Xian Zhao \\ and Xun Sun*
}

The formation energies, charge transition levels, and electronic structures of positively charged, neutral, and negatively charged hydrogen vacancies in the $\mathrm{NH}_{4} \mathrm{H}_{2} \mathrm{PO}_{4}$ (ADP) crystal are investigated in the framework of density functional theory with local and hybrid exchange-correlation functionals. The inclusion of nonlocal exchange opens the ADP fundamental band gap by nearly $1 \mathrm{eV}$ and well reproduces the experimental value. The van der Waals (vdW) interaction is found to have a major influence on the energetics of charged hydrogen vacancies in ADP. The calculated relative stability of $\mathrm{V}_{\mathrm{H}}^{+}$and $\mathrm{V}_{\mathrm{H}}^{-}$with vdW interaction could well explain the break point on the measured conductivity curve of the ADP crystal in the high temperature region. On the other hand, a missing $\mathrm{H}$ atom in the $\left(\mathrm{H}_{2} \mathrm{PO}_{4}\right)^{-}$group is found to be more energetically preferable than $\mathrm{NH}_{4}{ }^{+}$. It could capture a hole carrier to form a molecular-type polaron with its adjacent two $\mathrm{O}$ atoms, and be responsible for the optical absorption under irradiation by a high-intensity laser beam.

Received 11th December 2017

Accepted 2nd February 2018

DOI: 10.1039/c7ra13212c

rsc.li/rsc-advances

and physical properties of ADP crystals under ideal environment. For instance, the morphology of ADP crystals were modelled based on the calculation of chemical bond strength and the interaction between crystal surfaces. ${ }^{17,18}$ The density functional theory (DFT) calculation was carried out to present the microscopic mechanism of antiferroelectricity in ADP crystal. ${ }^{19}$

It is noted that the LDT of ADP could be reduced due to the existence of intrinsic point defects in the crystal. Many experimental efforts have been made to detect the defects in the crystal. In Murphy's research, there is an inflection point on the conductivity curve between the low and high temperature areas, which can be used to characterize the formation energy and diffusion of the defect. ${ }^{20}$ As Pollock didn't find such inflection point, he proposed that the defect formation and diffusion is a process in ADP crystal. ${ }^{21}$ Later, Harris proved that the proton vacancy in $\mathrm{NH}_{4}{ }^{+}$contributes to the electric conduction of ADP. ${ }^{22}$ Rath further illustrated the impact of impurity and hydrogen vacancy on the conductivity and dielectric loss of doped ADP crystals. ${ }^{23}$ Also, Abdel-Kader regarded the inflection point as the relative rotary movement of phosphate group with its surrounding hydrogen proton. ${ }^{24}$ Therefore, hydrogen vacancy plays a key role in the physical properties of ADP such as electrical conductivity, dielectric loss, etc. Furthermore, the hydrogen vacancy could introduce impurity absorption that will reduce the optical quality of the crystal. It could also trap electron or hole carriers to bring out the change of electronic and optical properties. However, the influence of hydrogen vacancy defect on the physical properties of ADP crystals is still in dispute in experiment. The related theoretical studies have not been reported yet. Studying the structure and properties of 
hydrogen vacancy defect in ADP could help us to understand the microcosmic mechanism of defect induced conductivity and optical damage.

In this paper, we investigated the relative stability and electronic structures of charged hydrogen vacancy defects in ADP crystal by using hybrid DFT. The influence of supercell size, the electron exchange and correlation functional, and van der Waals (vdW) interaction on the defect formation energies of charged hydrogen vacancies are carefully examined in order to guarantee the accuracy of calculation results. Hybrid DFT was used to obtain more reliable description of defect formation energies, defect levels, and the localization of the electron distribution than (semi)local generalized gradient approximation (GGA) functional in wide-band-gap semiconductors ${ }^{25-30}$ by introducing some amount of exact exchange from Hartree-Fock theory in the electron exchange and correlation functional. The vdW interaction is found to be very important in describing the formation energies and defect levels for ADP crystal.

\section{Computational method}

The Vienna $A b$ Initio Simulation Package (VASP) ${ }^{31,32}$ based on the first-principles and the projector-augmented-wave (PAW) potential is implemented in the present calculation. Thereby the $\mathrm{N} 2 \mathrm{~s}^{2} 2 \mathrm{p}^{3}, \mathrm{H} 1 \mathrm{~s}^{1}, \mathrm{P} 3 \mathrm{~s}^{2} 3 \mathrm{p}^{3}$, and $\mathrm{O} 2 \mathrm{~s}^{2} 2 \mathrm{p}^{4}$ states are treated as valence electrons. The general gradient approximation (GGA) of Perdew Burke and Ernzerhof $(\mathrm{PBE})^{33}$ is employed to optimize the crystal structure with the force convergence criterion of $0.01 \mathrm{eV} \AA^{-1}$. The energetics and electronic structures are calculated by the screened-exchange hybrid density functional of Heyd, Scuseria, and Ernzerhof HSE06 (ref. 34 and 35). In this approach, the long-range exchange potential and the correlation potential are calculated with PBE functional, while the short-range exchange potential is calculated by mixing a fraction of nonlocal Hartree-Fock exchange with PBE. The screening length and mixing parameter are fixed at $10 \AA$ and 0.25 respectively. To confirm the convergence of the calculations, the dependence of the total energy on the $k$-point set mesh $^{37}$ and cutoff energy of bulk ADP is investigated with PBE and HSE06 respectively. ${ }^{36}$ According to the convergence test, the cutoff energy is set to $400 \mathrm{eV}$ for both PBE and HSE06 calculations, and the $k$-point sets are chosen to $4 \times 4 \times 4$ and $2 \times 2 \times 2$ for PBE and HSE06 calculations respectively. Another two types of hybrid functionals HSE03 (ref. 38) and PBE0 (ref. 39 and 40) are also used to test the performance of hybrid functionals on the defect formation energy and the electronic properties. The supercell containing 48 atoms are used to construct the defect models. All the atoms and the lattice constants are fully relaxed by using the conjugate gradient techniques. DFT/vdW-WF2 method $^{\mathbf{4 1 - 4 3}}$ is used to consider the hydrogen bond interaction in the whole system. The tetragonal supercell with four $\mathrm{NH}_{4} \mathrm{H}_{2} \mathrm{PO}_{4}$ units (48 atoms) is used to model hydrogen vacancy defects in the crystal with defect density of 2.08 atom\%. An electron is removed or added to the neutral system of hydrogen vacancy $\left(\mathrm{V}_{\mathrm{H}}^{0}\right)$ in order to model the positively $\left(\mathrm{V}_{\mathrm{H}}^{+}\right)$and negatively $\left(\mathrm{V}_{\mathrm{H}}^{-}\right)$charged hydrogen vacancy structure. The charged systems are also fully relaxed. The lattice vectors are refined as $\mathbf{A}$
Table 1 The chemical potentials of $\mathrm{H}$ with different DFT functionals and van der Waals interaction

\begin{tabular}{ll}
\hline Functionals & The chemical potential of $\mathrm{H}(\mathrm{eV})$ \\
\hline PBE & -3.46 \\
HSE06 & -3.82 \\
HSE03 & -3.82 \\
PBE0 & -3.95 \\
PBE + vdW & -3.34 \\
HSE06 + vdW & -3.82
\end{tabular}

$=(a \mathbf{i}+a \mathbf{j}), \mathbf{B}=(a \mathbf{i}-a \mathbf{j}), \mathbf{C}=c \mathbf{k}$ with the lattice constants of conventional unit cell $a=b=7.50 \AA$, and $c=7.55 \AA^{44,45}$

The defect formation energies $E_{\mathrm{f}}$ of defect $X$ with charge state $q$ dependent on the Fermi level position is defined as $^{\mathbf{4 6 - 4 8}}$

$$
\begin{aligned}
E_{\mathrm{f}}\left(X^{q}\right)= & E^{\mathrm{tot}}\left(X^{q}\right)-E^{\mathrm{tot}}(\text { pristine })+\sum_{i} n_{i} \mu_{i} \\
& +q\left(E_{\mathrm{F}}+E_{\mathrm{v}}+\Delta V\right),
\end{aligned}
$$

where $E^{\text {tot }}\left(X^{q}\right)$ is the total energy of a supercell with defects. $E^{\text {tot }}$ (pristine) is the total energy of the supercell without any defects. $n_{i}$ is the species number of the atoms which is removed or added to the supercell. $\mu_{i}$ is the chemical potential of the element $i . E_{\mathrm{F}}$ is the Fermi energy with respect to the valenceband maximum (VBM) $E_{\mathrm{v}}$ in the pristine single crystal. $\Delta V$ is the difference between the electrostatic potentials of the defective and pristine systems. The chemical potential of $\mathrm{H}$ is calculated by putting a $\mathrm{H}_{2}$ molecule in a cubic cell with lattice constant of $10.00 \AA$. The values calculated by many considered functionals and those considered van der Waals interactions are listed in Table 1. It is seen that the calculation values of $\mathrm{H}$ chemical potential mainly depend on the chosen of the type of the electron exchange-correlation potentials. The traditional PBE functional always overestimates the energy of $\mathrm{H}$ as compared to the hybrid functionals.

The thermodynamic transition levels $\varepsilon\left(q_{1} / q_{2}\right)$ is defined as the Fermi-level position for which the formation energies of charge states $q_{1}$ and $q_{2}$ are equal: ${ }^{46,49}$

$$
\varepsilon\left(q_{1} / q_{2}\right)=\frac{E_{\mathrm{f}}\left(X^{q_{1}}\right)-E_{\mathrm{f}}\left(X^{q_{2}}\right)}{q_{2}-q_{1}}
$$

where $E_{\mathrm{f}}\left(X^{q}\right)$ is the formation energy of the defect $X$ in the charge state $q$. The experimental significance of the charge transition level is that for Fermi-level position below $\varepsilon\left(q_{1} / q_{2}\right)$, charge state $q_{1}$ is stable, while for Fermi-level position above $\varepsilon\left(q_{1} / q_{2}\right)$, charge state $q_{2}$ is stable.

\section{Results and discussion}

\subsection{The structure of hydrogen vacancies in ADP}

We construct the hydrogen vacancy $\left(\mathrm{V}_{\mathrm{H}}\right)$ model by removing one hydrogen atom from the ADP supercell with 48 atoms. Firstly, we compare accuracy of considered electron exchangecorrelation functionals on the crystal structure prediction by comparing the calculated the lattice constants of $a, b$, and $c$ of ADP with experimental data. ${ }^{50,51}$ We then plot the 
corresponding mean absolute error (in \%) in Fig. 1. We can see that HSE03 and PBE0 functionals show larger errors than PBE and HSE06 functionals for both $a$ and $c$ lattice parameters. PBE and HSE06 functionals show the similar extent on the prediction of lattice parameters. Therefore, we choose PBE functional to optimize the lattice constant and atomic position in order to save the computational cost. The optimized lattice constants by PBE are $a=b=7.32 \AA$ and $c=7.42 \AA$ with deviation of $2.40 \%$ and $1.72 \%$ with respect to the experimental values of $a=b=7.50 \AA$ and $c=7.55 \AA^{44,45}$

Fig. 2(a) shows the ADP single crystal grown by the temperature reduction method, and its corresponding crystal structure is shown in Fig. 2(b). The ADP crystal structure is tetragonal with $I \overline{4} 2 d$ space group. It is seen from Fig. 2(b) that each $\mathrm{NH}_{4}{ }^{+}$group is connected with $\operatorname{six}\left(\mathrm{H}_{2} \mathrm{PO}_{4}\right)^{-}$groups via covalent bond of $\mathrm{N}-\mathrm{H}-\mathrm{O}$, while each $\left(\mathrm{H}_{2} \mathrm{PO}_{4}\right)^{-}$group bonds with four $\left(\mathrm{H}_{2} \mathrm{PO}_{4}\right)^{-}$groups by the $\mathrm{P}-\mathrm{O}-\mathrm{H} \cdots \mathrm{O}$ hydrogen bond. ${ }^{19}$ Therefore, there are two kinds of $\mathrm{H}$ atoms in the ADP crystal $-\mathrm{H}_{1}$ in the $\left(\mathrm{H}_{2} \mathrm{PO}_{4}\right)^{-}$group with the fractional coordinate $(0.750,0.350,0.375)$ and $\mathrm{H}_{2}$ in the $\mathrm{NH}_{4}{ }^{+}$ group with the fractional coordinate $(0.500,0.411,0.937)$, as labelled in Fig. 2(c) and (d). It is found that $\mathrm{V}_{\mathrm{H}_{1}}$ leads to small amount of lattice relaxation whereas $\mathrm{V}_{\mathrm{H}_{2}}$ defect causes large atomic distortion regarding to the change of atomic location, bonding length, bonding angle and symmetry of $\mathrm{NH}_{4}{ }^{+}$group. In order to accurately obtain the relative stability of $\mathrm{V}_{\mathrm{H}_{1}}$ and $\mathrm{V}_{\mathrm{H}_{2}}$, we compare the calculated defect formation energies (DFEs) of neutral $\mathrm{V}_{\mathrm{H}_{1}}$ and $\mathrm{V}_{\mathrm{H}_{2}}$ with different functionals and listed in Table 2. It is seen that although the absolute values of DFEs of $\mathrm{V}_{\mathrm{H}_{1}}$ and $\mathrm{V}_{\mathrm{H}_{2}}$ varies for different functionals, the DFE of $\mathrm{V}_{\mathrm{H}_{1}}$ is always lower than that of $\mathrm{V}_{\mathrm{H}_{2}}$ indicating that $\mathrm{V}_{\mathrm{H}_{1}}$ is energetically preferable to form in ADP crystal. The easier missing of $\mathrm{H}_{1}$ atom is mainly due to the weak hydrogen bond interaction. Therefore, we studied the

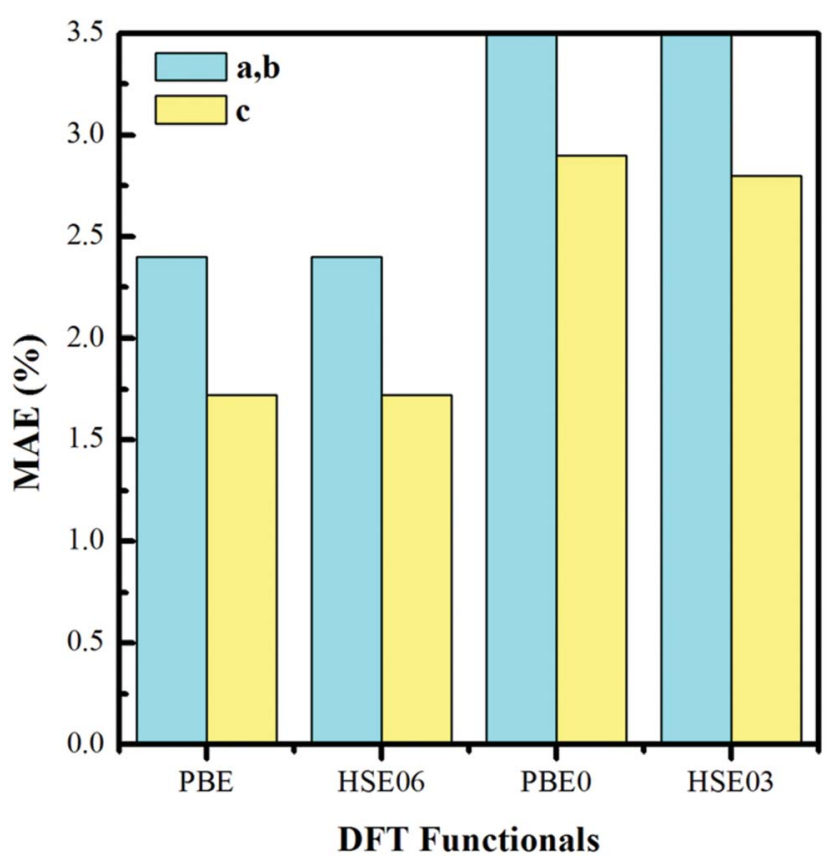

Fig. 1 Mean absolute error (in \%) on the optimized lattice constants of bulk ADP as compared with the experimental values. charged $\mathrm{V}_{\mathrm{H}}$ defects based on the $\mathrm{V}_{\mathrm{H}_{1}}$ model. The model of positively charged $\mathrm{V}_{\mathrm{H}}^{+}$is constructed by removing one electron from the neutral $\mathrm{V}_{\mathrm{H}}$ system, and that of negatively charged $\mathrm{V}_{\mathrm{H}}^{-}$is constructed by adding one electron to the neutral $\mathrm{V}_{\mathrm{H}}$ system. The optimized structures are shown in Fig. 2(e) and (f). It is seen that the distance of $\mathrm{O}_{1}$ and $\mathrm{O}_{2}$ adjacent to the $\mathrm{V}_{\mathrm{H}}^{+}$is dramatically shortened by $37.70 \%$ from 2.387 to $1.488 \AA$ (Table 3 ) as compared to the case of neutral $V_{H}$ defect due to the capture of hole, showing strong interaction of $\mathrm{O}_{1}$ and $\mathrm{O}_{2}$. In this case, the distance of $\mathrm{O}_{1}-\mathrm{O}_{2}$ is similar to the bond length of $1.49 \AA$ for the neutral peroxyl bridge in vitreous silicon dioxide. ${ }^{52,53}$ The movement of $\mathrm{O}$ toward $\mathrm{V}_{\mathrm{H}}^{+}$defect leads to the elongation of $\mathrm{P}-\mathrm{O}$ bond by $9.08 \%$. On the contrary, the capture of an electron carrier by $\mathrm{V}_{\mathrm{H}}$ leads to the elongation of the $\mathrm{O}_{1}-\mathrm{O}_{2}$ distance by $20.74 \%$ (from 2.387 to $2.882 \AA$ ) due to the Coulomb repulsion of captured electron with the electrons around $\mathrm{O}_{1}$ and $\mathrm{O}_{2}$, indicating a much weak interaction of $\mathrm{O}_{1}$ and $\mathrm{O}_{2}$. The outward movement of $\mathrm{O}$ regarding to $\mathrm{V}_{\mathrm{H}}^{-}$only leads to the slight shortening of $\mathrm{P}-\mathrm{O}$ bond by $2.01 \%$, showing small influence on the $\mathrm{PO}_{4}{ }^{3-}$ group.

\subsection{Relative stability of hydrogen vacancies in ADP}

We investigate the relative stability of hydrogen vacancies in ADP crystal by calculating the defect formation energies dependent with the Fermi level and the charge transition levels. At the beginning, we examine the influence of calculation model and parameters on the accuracy of defect formation energies of charged point defects by taking $\mathrm{V}_{\mathrm{H}}^{-}$as an example. The charged defects always introduce finite-size error that arise from the electrostatic interaction between charged defects in neighboring cell images and the structural strain introduced by the defect due to the limited size of the supercell within periodic boundary condition. We therefore first compare the defect formation energies of $\mathrm{V}_{\mathrm{H}}^{-}$calculated in 48-atom and 384-atom supercells within PBE functional, as shown in Table 4. It is seen that the calculated defect formation energies of $\mathrm{V}_{\mathrm{H}}^{-}$in 384-atom supercell is only $0.04 \mathrm{eV}$ lower than that in 48 -atom supercell, indicating extremely slow energy change with the increase of supercell size until to infinite limit. Therefore, the finite-size error to the defect formation energies of charged $\mathrm{V}_{\mathrm{H}}$ could be neglected, and the smaller supercells (48 atoms) are used to investigate the energetic and electronic properties of $\mathrm{V}_{\mathrm{H}}$ in ADP crystal in order to save the computational cost. Second, we consider the influence of $\mathrm{vdW}$ interaction on the defect formation energy of $\mathrm{V}_{\mathrm{H}}^{-}$. The inclusion of vdW interaction by DFT/vdW-WF2 method has been proved to obtain more accurate description of the molecule-surface interaction and the system with hydrogen bond interaction. ${ }^{54-59}$ Here we compare the calculated defect formation energies of $\mathrm{V}_{\mathrm{H}}^{-}$with and without vdW correction within PBE functional, as shown in Table 4. It is seen that the vdW correction could reduce the defect formation energy of $\mathrm{V}_{\mathrm{H}}^{-}$by $0.24 \mathrm{eV}$, which is one order of magnitudes larger than the error of finite-size effect. Such energy difference may lead to the different result of the relative stability of charged $\mathrm{V}_{\mathrm{H}}$ in ADP crystal, and the vdW correction should thus be considered in the following calculations. Finally, we consider the effect of screened exchange potential by comparing the calculated 
(a)

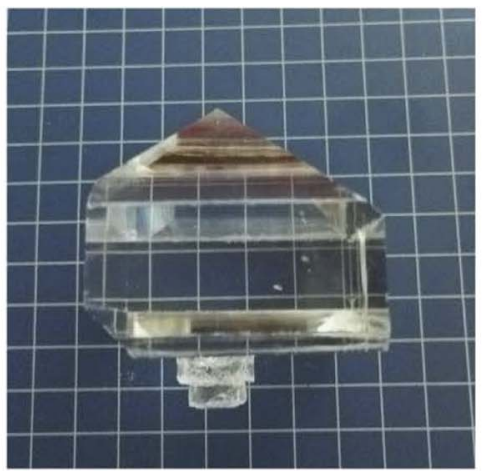

(c)

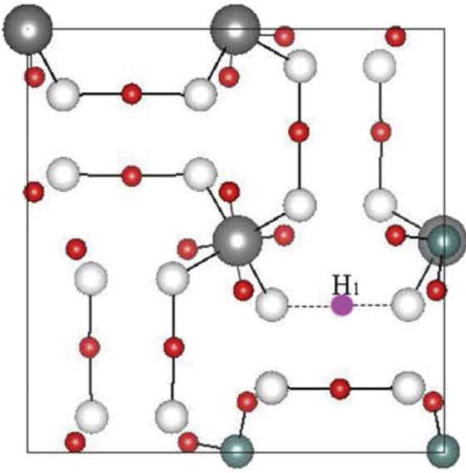

(e)

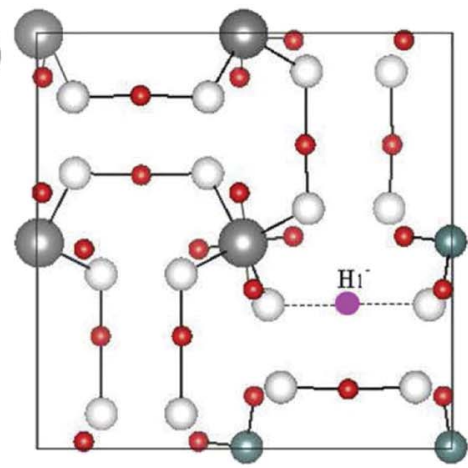

(b)
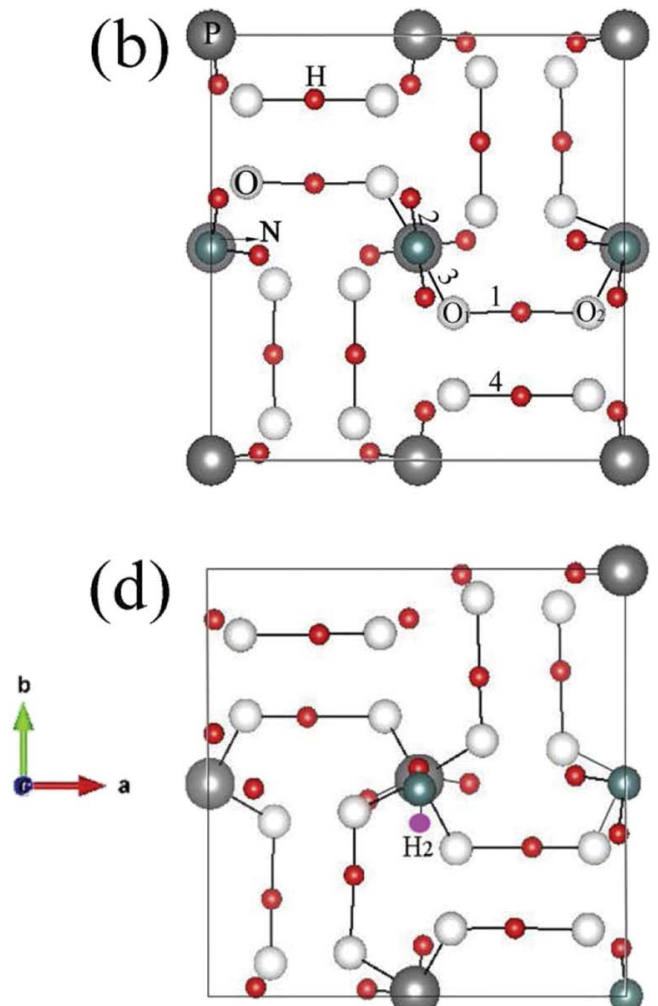

(f)
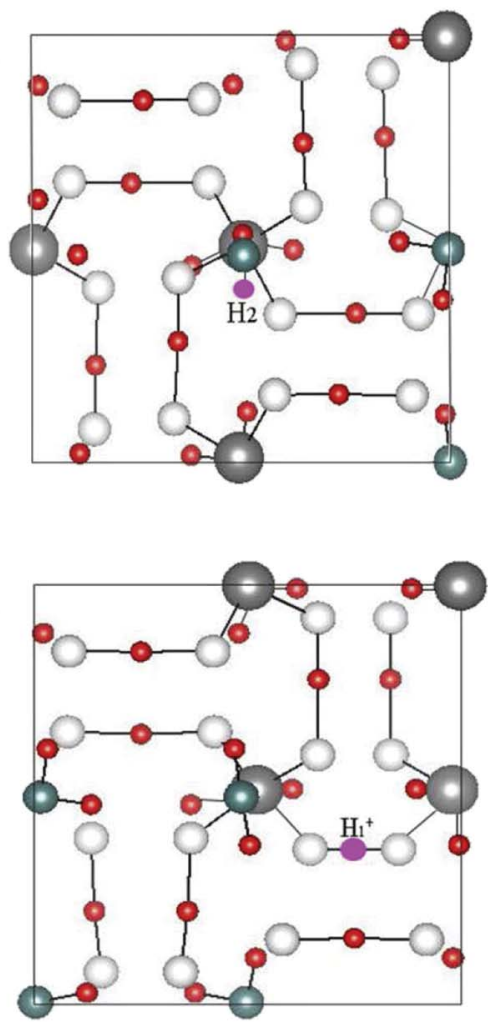

Fig. 2 ADP crystal grown with the temperature reduction method (a), top-view of the crystal structures of pristine ADP (b), ADP with neutral $H_{1}$ (c) and $\mathrm{H}_{2}$ (d) vacancies, as well as $\mathrm{H}_{1}$ vacancies with -1 (e) and +1 (f) charge states. The vacancies are indicated in the small rose red balls.

Table 2 The calculated defect formation energies of neutral $\mathrm{V}_{\mathrm{H}_{1}}$ and $\mathrm{V}_{\mathrm{H}_{2}}$ with different functionals

\begin{tabular}{|c|c|c|}
\hline \multirow[b]{2}{*}{ Functionals } & \multicolumn{2}{|c|}{ Defect formation energies $E_{\mathrm{f}}(\mathrm{eV})$} \\
\hline & $\mathrm{V}_{\mathrm{H}_{1}}$ & $\mathrm{~V}_{\mathrm{H}_{2}}$ \\
\hline PBE & 3.06 & 3.44 \\
\hline HSE06 & 4.56 & 4.70 \\
\hline PBE0 & 4.37 & 4.45 \\
\hline HSE03 & 3.94 & 4.19 \\
\hline
\end{tabular}

defect formation energies of $\mathrm{V}_{\mathrm{H}}^{-}$by PBE and HSE06. From the calculation results shown in Table 4 we can see that the HSE06 functional that mixing $25 \%$ nonlocal Hartree-Fock exchange
Table 3 Bond lengths and distance between atoms closed to the hydrogen vacancy as labelled in Fig. 2. The numbers of chemical bonds are related to those labelled in Fig. 2(b). The units are in $\AA$

\begin{tabular}{llllll}
\hline Bonds/distance & $\begin{array}{l}\text { Pristine } \\
\mathrm{ADP}\end{array}$ & $\mathrm{V}_{\mathrm{H}_{1}}$ & $\mathrm{~V}_{\mathrm{H}_{2}}$ & $\mathrm{~V}_{\mathrm{H}}^{-}$ & $\mathrm{V}_{\mathrm{H}}^{+}$ \\
\hline 1 & 1.200 & - & 1.343 & - & - \\
2 & 1.039 & 1.038 & - & 1.006 & 1.035 \\
3 & 1.551 & 1.542 & 1.528 & 1.501 & 1.682 \\
4 & 1.200 & 1.201 & 1128 & 1.207 & 1.195 \\
$\mathrm{O}_{1}-\mathrm{O}_{2}$ & 2.401 & 2.387 & 2.437 & 2.882 & 1.488
\end{tabular}

with PBE could largely enhance the defect formation energy of $\mathrm{V}_{\mathrm{H}}^{-}$by $3.35 \mathrm{eV}$. It is one order of magnitudes larger than the 
Table 4 The comparison of defect formation energies of $V_{H}$ in ADP when considering different defect location, supercell size, vdW correction, and hybrid electron exchange potential

\begin{tabular}{ll}
\hline Parameters & $\begin{array}{l}\text { Defect formation } \\
\text { energies } E_{\mathrm{f}}(\mathrm{eV})\end{array}$ \\
\hline $\mathrm{V}_{\mathrm{H}_{1}}$, HSE06, 48 atoms & 4.56 \\
$\mathrm{~V}_{\mathrm{H}_{2}}$, HSE06, 48 atoms & 4.70 \\
$\mathrm{~V}_{\mathrm{H}_{1}}^{-}$, PBE, 384 atoms & $3.16-E_{\mathrm{F}}$ \\
$\mathrm{V}_{\mathrm{H}_{1}}^{-}$, PBE, 48 atoms & $3.12-E_{\mathrm{F}}$ \\
$\mathrm{V}_{\mathrm{H}_{1}}^{-}$, PBE + vdW, 48 atoms & $2.88-E_{\mathrm{F}}$ \\
$\mathrm{V}_{\mathrm{H}_{1}}^{-}$, HSE06 + vdW, 48 atoms & $4.33-E_{\mathrm{F}}$ \\
\hline
\end{tabular}

correction of vdW interaction, and should not be neglected. As HSE06 has been proved to better describe the defect formation energy, electron localization, and defect levels than GGA, HSE06 functional is used in the following calculations. ${ }^{60,61}$ Overall, the energetics and electronic structures of $\mathrm{V}_{\mathrm{H}}$ are calculated within HSE06 hybrid functional with vdW correction by using tetragonal ADP supercells containing 48 atoms after carefully parameter examination.

The calculated defect formation energies of $\mathrm{V}_{\mathrm{H}}^{+}, \mathrm{V}_{\mathrm{H}}^{0}$, and $\mathrm{V}_{\mathrm{H}}^{-}$ with and without vdW correction are compared in Fig. 3. We found that the vdW interaction play a key role in the calculation of defect formation energies and charge transition levels. For example, the vdW correction largely shifts the charge transition level $\varepsilon(+/-)$ toward the valence band maximum (VBM) from $2.93 \mathrm{eV}$ to $1.12 \mathrm{eV}$. We could get different conclusions according to this result: without the consideration of vdW interaction, the positively charged hydrogen vacancy $\mathrm{V}_{\mathrm{H}}^{+}$is the most stable defect in majority of ADP crystals, and the negatively charged hydrogen vacancy $\mathrm{V}_{\mathrm{H}}^{-}$only exists in near-stoichiometric crystal; whereas $\mathrm{V}_{\mathrm{H}}^{-}$is more stable than $\mathrm{V}_{\mathrm{H}}^{+}$when the Fermi level moves up to $1.12 \mathrm{eV}$ if the vdW

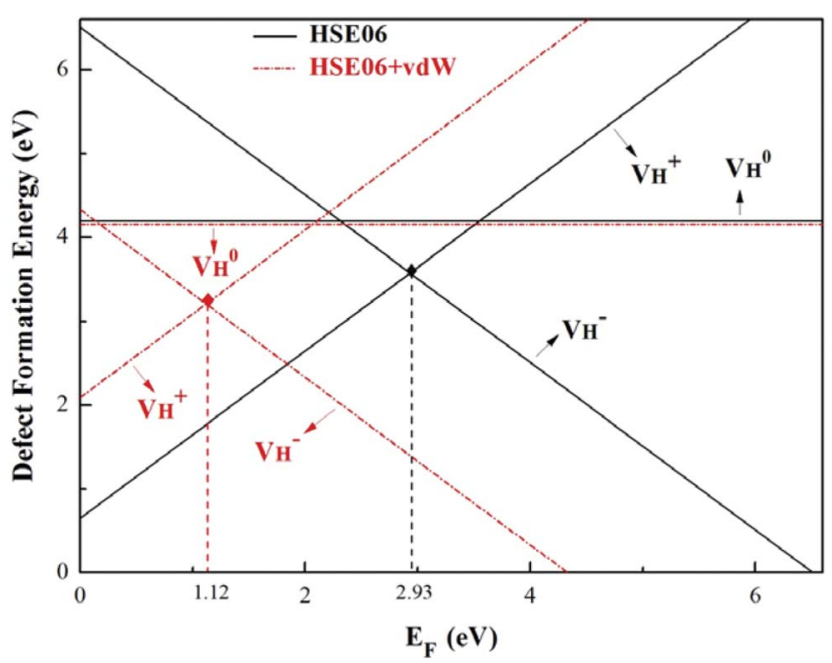

Fig. 3 Defect formation energies of $V_{H}$ with positive, neutral, and negative charge states as a function of the Fermi level for HSEO6 and HSEO6 + vdW methods. The range of Fermi energy corresponds to the calculated fundamental band gap of $6.76 \mathrm{eV}$ for pristine ADP crystal. interaction is considered. The calculation results with vdW interaction are for sure more accurate than those without the vdW interaction. We thus only discuss the calculation results with vdW interaction. It is seen that $\mathrm{V}_{\mathrm{H}}^{+}$is more stable than $\mathrm{V}_{\mathrm{H}}^{0}$ and $\mathrm{V}_{\mathrm{H}}^{-}$when the Fermi level lies near the $\mathrm{VBM}$ while $\mathrm{V}_{\mathrm{H}}^{-}$is energetically preferable when the Fermi level lies higher than $1.12 \mathrm{eV}$. As in ADP crystals, $\mathrm{H}$ atoms are much easier to be missed than $\mathrm{N}, \mathrm{P}$, and $\mathrm{O}$ atoms to form $\mathrm{V}_{\mathrm{H}}$ defects, the Fermi level could lies below the middle of the electronic band gap of ADP. Therefore, in this region, $\mathrm{V}_{\mathrm{H}}^{+}$and $\mathrm{V}_{\mathrm{H}}^{-}$are all the possible defects in practical ADP crystals. The results demonstrate that the neutral $\mathrm{V}_{\mathrm{H}}^{0}$ is instable in the ADP crystal, which is similar to the case in $\mathrm{SiO}_{2} \cdot{ }^{62}$ The instability of the neutral $\mathrm{V}_{\mathrm{H}}^{0}$ defect indicates that the hydrogen vacancies in majority of ADP crystals prefer to trap hole or electron carriers. There are two extreme situations: when the defect concentration of hydrogen vacancies is high, $\mathrm{V}_{\mathrm{H}}$ defects prefer to capture hole carriers and act as acceptor centers; if the grown ADP crystal is with good quality (very small concentration of $\mathrm{V}_{\mathrm{H}}$ ), these hydrogen vacancies mainly prefer to capture electron carriers and act as donor centers. If the situation is in between, part of $\mathrm{V}_{\mathrm{H}}$ may capture holes and the rest captures electrons which results in the spatial charge separation in ADP crystals. These results may give a reasonable explanation regarding to the experimentally observed inflection point in the conductivity curve from the aspect of defect behavior. ${ }^{63}$ At the low temperature region, the concentration of hydrogen vacancies is very low, and the amount of the electron carriers in the crystals is thus also small. In this case, $\mathrm{V}_{\mathrm{H}}^{-}$is energetically preferable and therefore, the neutral hydrogen vacancy defects prefer to capture electrons to form stable $\mathrm{V}_{\mathrm{H}}^{-}$defects. It further reduces the concentration of electron carriers, and mainly contributes to the low conductivity of ADP crystal. With the increase of temperature, more hydrogen atoms could escape from their original lattice sites due to the thermal vibration, leaving a large amount of hydrogen vacancies in the lattice. On the one hand, it will largely increase the concentration of electron carriers in the crystal. On the other hand, with the increase of hydrogen vacancy concentration, a large part of the vacancy defects prefer to capture hole carriers instead of electron carriers to form stable $\mathrm{V}_{\mathrm{H}}^{+}$defects, which leaves much more electrons in the crystal, leading to the sharp increase of the conductivity of the ADP crystal. The well explanation of the measured conductivity phenomenon further proved the importance of considering the vdW interaction in ADP calculations.

\subsection{Electronic structure of hydrogen vacancies in ADP}

In order to better understand the electron/hole trapping behaviors and the defect induced impurity states in the band gap of ADP crystal, we calculated the density of states of stable hydrogen vacancies and the electron distributions around the hydrogen vacancy defects in ADP crystal. First, we compare the calculated electronic band gaps of ADP by different functionals. As shown in Table 5, HSE06 functional shows the best description of band gap of ADP crystal $(6.76 \mathrm{eV})$ as compared to 
Table 5 The band gap of the pristine ADP crystal with different DFT functionals

\begin{tabular}{ll}
\hline Functionals & The band gap $(\mathrm{eV})$ \\
\hline Experimental & 6.96 \\
PBE & 5.97 \\
HSE06 & 6.76 \\
PBE0 & 7.79 \\
HSE03 & 6.51
\end{tabular}

the experiment value $6.96 \mathrm{eV},{ }^{64}$ indicating that HSE06 could well reproduce the electronic properties such as density of states, electron distribution of this system. The local PBE functional could underestimate the band gap as usual, and the PBE0 functional is proved to overestimate the band gap compared with HSE06, which has been reported in the literature. ${ }^{\mathbf{5 0 5 1}}$ The HSE03 functional shows fewer consistence with experiment than HSE06. Also, we found that the consideration of vdW correction could not change the calculated band gap of ADP, showing little influence on the electronic structures of ADP crystal.

The calculated partial density of states (PDOS) of neutral, positively and negatively charged hydrogen vacancies as compared to those of pristine ADP crystal are shown in Fig. 4. It is seen that the VBM of pristine ADP is mainly derived from $\mathrm{O} 2 \mathrm{p}$ states while the conduction band minimum (CBM) comes from the mixing of $\mathrm{H} 1 \mathrm{~s}, \mathrm{O} 2 \mathrm{p}$, and $\mathrm{P} 3 \mathrm{p}$ states. The formation

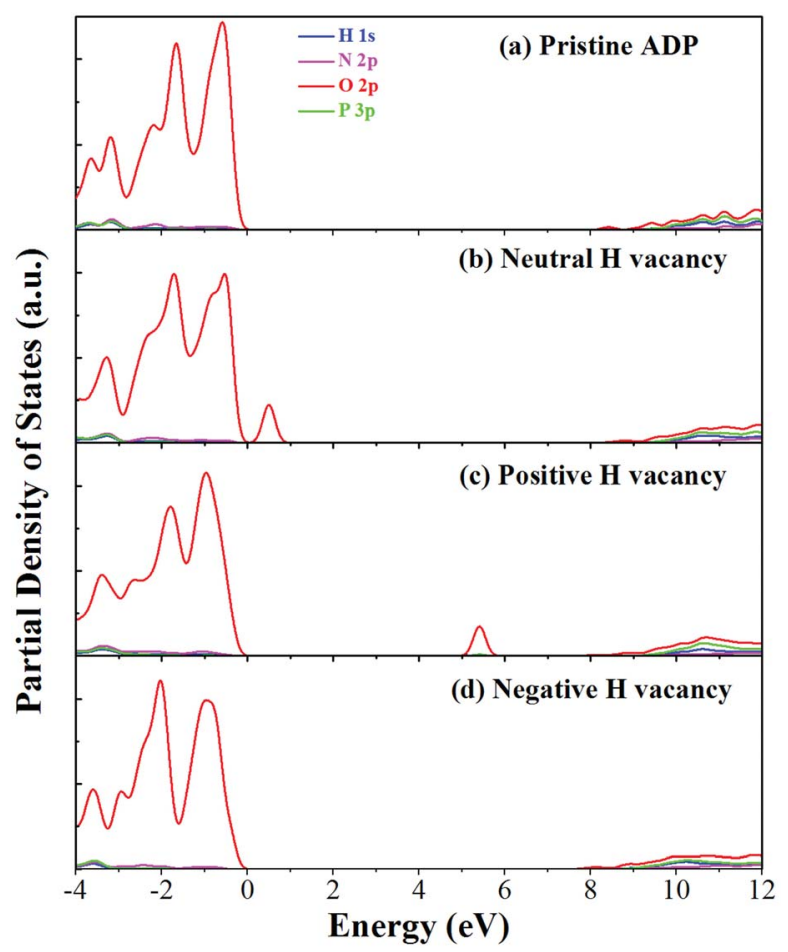

Fig. 4 PDOS of $\mathrm{H}, \mathrm{N}, \mathrm{O}$, and $\mathrm{P}$ in pristine ADP (a) and crystal with neutral (b), positively (c) and (d) negatively charged hydrogen vacancies. of a neutral $\mathrm{V}_{\mathrm{H}}^{0}$ defect introduces a half-occupied defect state at about $0.5 \mathrm{eV}$ above the VBM in the band gap (Fig. 4b). This defect state is derived from $\mathrm{O} 2 \mathrm{p}$ states of its neighboring $\mathrm{O}$ atoms (Fig. 5a), which is similar to the results of electronic properties calculations for hydrogen vacancy in silica. ${ }^{62}$ It could introduce weak optical absorption just near the absorption edge of pristine ADP crystal that comes from the electron transition from the defect state to the CBM and may hardly be recognized.

When the neutral $\mathrm{V}_{\mathrm{H}}$ captures one hole to form $\mathrm{V}_{\mathrm{H}}^{+}$defect, the defect state in the band gap shifts toward CBM by $4.7 \mathrm{eV}$ and the band gap is reduced to $5.2 \mathrm{eV}$. This defect leads to the stronger coupling interaction of its adjacent $\mathrm{O}$ atoms, and finally forms O-O peroxyl bridge which is similar to the phenomenon of the positive hydrogen vacancy in KDP and the oxygen atom in a peroxyl bridge in silica. ${ }^{52,65}$ Finally, the hydrogen vacancy could capture a hole with its two adjacent $\mathrm{O}$ atoms to form a molecular-type polaron. The contributions of the adjacent $\mathrm{O}$ atoms and the other adjacent atoms have been plotted in Fig. $5 \mathrm{~b}$ and 6 respectively. We can see that the defect state of $\mathrm{V}_{\mathrm{H}}^{+}$comes from the mixing of $2 \mathrm{~s}$ and $2 \mathrm{p}$ states of its two neighboring $\mathrm{O}$ atoms that could also be confirmed from the charge density difference plot in Fig. 7a. In the above discussion, we found that the $\mathrm{P}-\mathrm{O}$ bond is stretched and the $\mathrm{PO}_{4}{ }^{3-}$ group also moves toward the $\mathrm{V}_{\mathrm{H}}^{+}$due to the formation of $\mathrm{O}-\mathrm{O}$ peroxyl bridge. Therefore, there is also a small amount of contribution of $\mathrm{P} 3 \mathrm{p}$ state on the $\mathrm{V}_{\mathrm{H}}^{+}$defect state in the band gap (Fig. 6). The VBM is mainly from the $2 p$ states of the adjacent $O$ atoms.

Another case is that the neutral $\mathrm{V}_{\mathrm{H}}$ defect captures one electron to form $\mathrm{V}_{\mathrm{H}}^{-}$defect. In this case, the isolated defect state in the band gap is full-occupied and downshifted to the valence

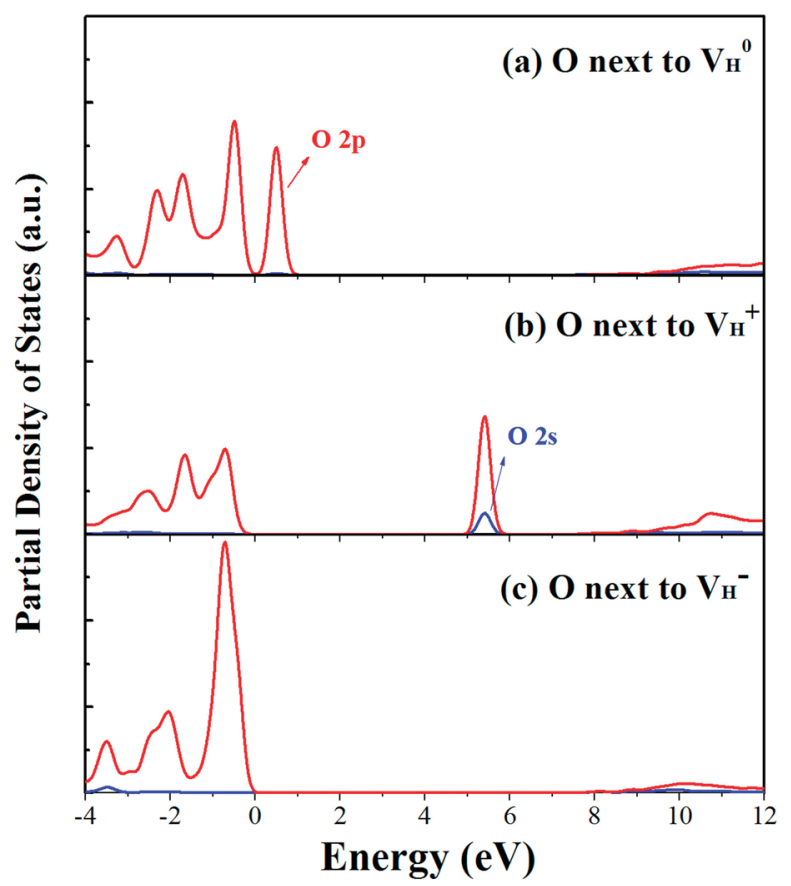

Fig. 5 PDOS of the $O$ atoms adjacent to the neutral (a), positive (b) and negative (c) $\mathrm{H}$ vacancies. 


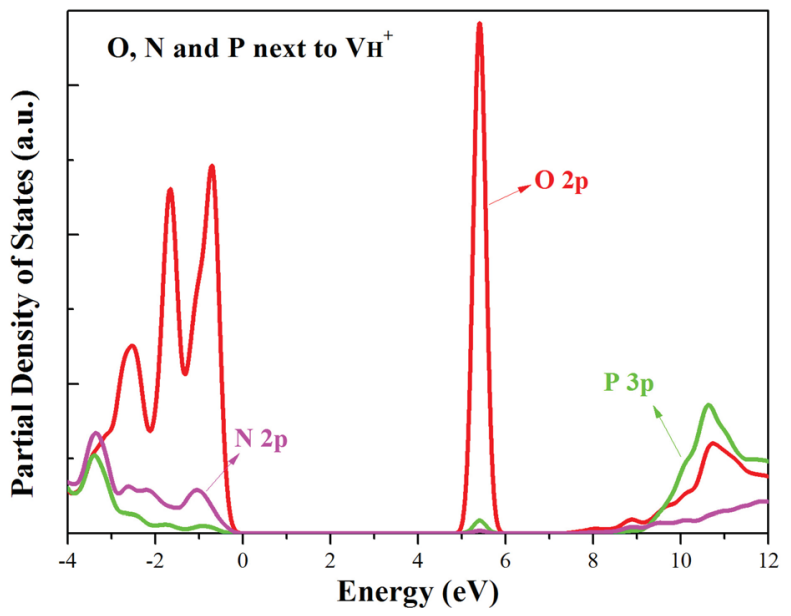

Fig. 6 PDOS of the $\mathrm{O}, \mathrm{N}$ and $\mathrm{P}$ atoms adjacent to the positive $\mathrm{H}$ vacancy.
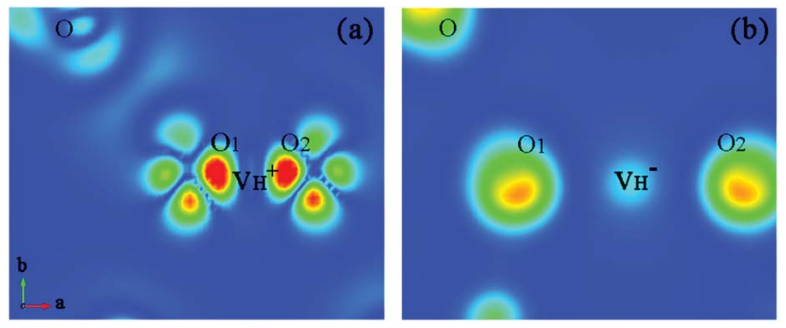

Fig. 7 The charge density difference contour maps for $\mathrm{V}_{H}^{+}$and $\mathrm{V}_{H}^{-}$. The regions from blue to red correspond to the electron density from 0 to 0.17 e $\AA^{-3}$.

band. At the same time, the valence bands are slightly upshifted due to the Coulomb repulsion of electrons. Therefore, the band gap of $\mathrm{V}_{\mathrm{H}}^{-}$defect is reduced to $6.27 \mathrm{eV}$, and the contribution of adjacent $\mathrm{O} 2 \mathrm{p}$ states to the $\mathrm{VBM}$ becomes much stronger (Fig. 5c). As compared to the $\mathrm{V}_{\mathrm{H}}^{+}$defect, the interaction between $\mathrm{O}_{1}$ and $\mathrm{O}_{2}$ becomes much weaker, while that of $\mathrm{P}$ and $\mathrm{O}$ becomes stronger. It could not introduce any new optical absorption for this defect.

By analyzing the electronic structures of the hydrogen vacancies, we get that only the positive charged hydrogen vacancy $\mathrm{V}_{\mathrm{H}}^{+}$is stable and could introduce additional optical absorption with respect to the intrinsic optical absorption. According to the calculation results, the new optical absorption introduced by $\mathrm{V}_{\mathrm{H}}^{+}$ could appear at about $260 \mathrm{~nm}$. The meaning of the stability of $\mathrm{V}_{\mathrm{H}}^{+}$ is that when the defect concentration is high, the additional optical absorption would be strengthened and easily detected by experimental measurement. ${ }^{66}$ They could also induce the precursor to absorb the laser energy, leading to the material damage and thus decreasing the laser damage threshold of ADP. Therefore, the concentration of positively charged hydrogen vacancy defects is suggested to be largely reduced during the growth of the crystal. In our experiment, we also observe the optical absorption at $355 \mathrm{~nm}(3.49 \mathrm{eV})$ that has been detected in many other experiments, such as ref. 3 . It is not expected to come from the intrinsic $\mathrm{H}$ vacancy as the calculated transition energy is not consistent with the measured absorption energy (more than $1 \mathrm{eV}$ difference). As we have tested the validity of local PBE functional and many types of hybrid functionals on the description of crystal structure and electronic properties of ADP crystal, the large energy difference of $1 \mathrm{eV}$ could not be from the calculation error. We infer that the optical absorption at $355 \mathrm{~nm}$ could come from the extrinsic impurity centers in ADP crystal, which should be intensively investigated later.

\section{Conclusions}

In conclusion, the density functional theory with (semi)local GGA and hybrid HSE06 exchange-correlation functionals were carried out to investigate the structures, relative stability, and electronic structures of the neutral and charged hydrogen vacancies in ADP crystals. The influence of supercell size, van der Waals interaction, and the screened exchange potential on the defect formation energies of charged hydrogen vacancies is carefully examined firstly. The calculation results show that the van der Waals interaction and the screened exchange potential have major effect on the calculation accuracy of both energetics and electronic structures, and thus should be considered. By comparing the defect formation energies of neutral $\mathrm{V}_{\mathrm{H}}$ in $\left(\mathrm{H}_{2} \mathrm{PO}_{4}\right)^{-}$and $\mathrm{NH}_{4}{ }^{+}$groups, we found that the $\mathrm{H}$ atom in the $\left(\mathrm{H}_{2} \mathrm{PO}_{4}\right)^{-}$group is easier to be missed to form $\mathrm{V}_{\mathrm{H}}$ defect. However, the neutral $\mathrm{V}_{\mathrm{H}}$ is unstable in the ADP crystals. It could capture a hole carrier to form its positive charge state or capture an electron to form its negative charge state in majority of ADP crystals. For the $\mathrm{V}_{\mathrm{H}}^{+}$defect, the hole is trapped around the two $\mathrm{O}$ atoms adjacent to the hydrogen vacancy to form a moleculartype polaron. We infer that the transition of $\mathrm{V}_{\mathrm{H}}^{-}$to $\mathrm{V}_{\mathrm{H}}^{+}$with the increase of temperature may be the dominant reason for the break point of the conductivity of ADP crystal in the high temperature region. On the other hand, $\mathrm{V}_{\mathrm{H}}^{+}$defect introduces a deep defect state in the middle of band gap. It could act as an optical absorption center in experiment and lead to the special optical absorption phenomenon and optical damage under irradiation by high-intensity laser beam.

\section{Conflicts of interest}

There are no conflicts to declare.

\section{Acknowledgements}

Thanks to the support of Shandong University and Institute of Crystal Materials Shandong University. This work was supported by the National Natural Science Foundation of China (Grant No. 51323002 and 51402173); and the Ministry of Education (Grant No. 625010360).

\section{References}

1 L. N. Rashkovich, KDP-family single crystals, Published under the Adam Hilger imprint by IOP Publishing Ltd, 1991. 
2 D. N. Nikogosyan, Nonlinear optical crystals: a complete survey, Springer, Cork, Ireland, 2001.

3 S. H. Ji, F. Wang, L. L. Zhu, X. G. Xu, Z. Wang and X. Sun, Noncritical phase-matching fourth harmonic generation of a 1053-nm laser in an ADP crystal, Sci. Rep., 2013, 3, 1605.

4 Y. F. Lian, L. L. Zhu, T. T. Sui, G. W. Yu, L. S. Zhang, H. L. Zhou, M. X. Xu and X. Sun, The rapid growth of ADP single crystal, CrystEngComm, 2016, 18, 7530-7536.

5 P. Bennema, Analysis of crystal growth models for slightly supersaturated solutions, J. Cryst. Growth, 1967, 1, 278-286.

6 R. J. Davey and J. W. Mullin, Growth of the $\{101\}$ faces of ammonium dihydrogen phosphate crystals in the presence of ionic species, J. Cryst. Growth, 1974, 23, 89-94.

7 R. J. Davey and J. W. Mullin, The effect of supersaturation on growth features on the $\{100\}$ faces of ammonium dihydrogen phosphate crystals, J. Cryst. Growth, 1975, 29, 45-48.

8 C. Belouet, E. Dunia and J. F. Petroff, X-ray topographic study of defects in $\mathrm{KH}_{2} \mathrm{PO}_{4}$ single crystals and their relation with impurity segregation, J. Cryst. Growth, 1974, 23, 243-252.

9 S. E. Božin and B. Žižić, Dependence of the linear growth rate of individual ammonium dihydrogen phosphate microcrystals on temperature, J. Cryst. Growth, 1981, 52, 820-823.

10 R. J. Davey, R. I. Ristić and B. Žižić, The role of dislocations in the growth of ammonium dihydrogen phosphate crystals from aqueous solutions, J. Cryst. Growth, 1979, 47, 1-4.

11 F. Lefaucheux, M. C. Robert and E. Manghi, A comparison between gel grown and solution grown crystals-case of ADP and KDP, J. Cryst. Growth, 1982, 56, 141-150.

12 P. V. Dhanaraj, G. Bhagavannarayana and N. P. Rajesh, Effect of amino acid additives on crystal growth parameters and properties of ammonium dihydrogen orthophosphate crystals, Mater. Chem. Phys., 2008, 112, 490-495.

13 R. I. Ristic and J. N. Sherwood, The growth rate variation of the (100) faces of ADP crystals in the presence of manganese ions, J. Phys. D: Appl. Phys., 1991, 24, 171.

14 D. L. Xu and D. F. Xue, Chemical bond analysis of the crystal growth of KDP and ADP, J. Cryst. Growth, 2006, 286, 108-113.

15 X. Zhao, C. T. Sun, Y. F. Si, M. N. Liu and D. F. Xue, Crystallization behaviors of ferroelectric and piezoelectric materials, Mod. Phys. Lett. B, 2009, 23, 3809-3815.

16 C. T. Sun and D. F. Xue, Hydrogen bonding nature during ADP crystallization, J. Mol. Struct., 2014, 1059, 338-342.

17 D. L. Xu, D. F. Xue and H. Ratajczak, Morphology and structure studies of KDP and ADP crystallites in the water and ethanol solutions, J. Mol. Struct., 2005, 740, 37-45.

18 D. L. Xu and D. F. Xue, Chemical bond analysis of the crystal growth of KDP and ADP, J. Cryst. Growth, 2006, 286, 108-113.

19 J. Lasave, S. Koval, N. S. Dalal and R. L. Migoni, Origin of antiferroelectricity in $\mathrm{NH}_{4} \mathrm{H}_{2} \mathrm{PO}_{4}$ from first principles, Phys. Rev. Lett., 2007, 98, 267601.

20 E. J. Murphy, Conduction in Single Crystals of Ammonium Dihydrogen Phosphate, J. Appl. Phys., 1964, 35, 2609-2614.

21 J. M. Pollock and M. Sharan, Conduction and diffusion in crystals containing hydrogen bonds. II. ammonium dihydrogen phosphate, J. Chem. Phys., 1969, 51, 3604-3607.
22 L. B. Harris and G. J. Vella, Direct current conduction in ammonium and potassium dihydrogen phosphate, $J$. Chem. Phys., 1973, 58, 4550-4557.

23 J. K. Rath and S. Radhakrishna, Electrical conductivity and dielectric loss studies of $\mathrm{MoO}_{4}{ }^{2-}$-doped $\mathrm{NH}_{4} \mathrm{H}_{2} \mathrm{PO}_{4}$ single crystals, J. Mater. Sci. Lett., 1987, 6, 929-931.

24 A. Abdel-Kader, Electrical conductivity of ammonium dihydrogen phosphate crystals with regard to crystal structure and thermal analysis, J. Mater. Sci.: Mater. Electron., 1991, 2, 7-10.

25 F. Oba, A. Togo, I. Tanaka, J. Paier and G. Kresse, Defect energetics in ZnO: a hybrid Hartree-Fock density functional study, Phys. Rev. B: Condens. Matter Mater. Phys., 2008, 77, 245202.

26 P. Deák, B. Aradi, T. Frauenheim, E. Janzén and A. Gali, Accurate defect levels obtained from the HSE06 rangeseparated hybrid functional, Phys. Rev. B: Condens. Matter Mater. Phys., 2010, 81, 153203.

27 J. L. Lyons, A. Janotti and C. G. Van de Walle, Shallow versus deep nature of $\mathrm{Mg}$ acceptors in nitride semiconductors, Phys. Rev. Lett., 2012, 108, 156403.

28 J. Paier, M. Marsman, K. Hummer, G. Kresse, I. C. Gerber and J. G. Ángyán, Screened hybrid density functionals applied to solids, J. Chem. Phys., 2006, 124, 154709.

29 P. Ágoston, K. Albe, R. M. Nieminen and M. J. Puska, Intrinsic n-type behavior in transparent conducting oxides: a comparative hybrid-functional study of $\operatorname{In}_{2} \mathrm{O}_{3}, \mathrm{SnO}_{2}$, and ZnO, Phys. Rev. Lett., 2009, 103, 245501.

30 T. Shimada, T. Ueda, J. Wang and T. Kitamura, Hybrid Hartree-Fock density functional study of charged point defects in ferroelectric $\mathrm{PbTiO}_{3}$, Phys. Rev. B: Condens. Matter Mater. Phys., 2013, 87, 174111.

31 G. Kresse and J. Furthmüller, Efficiency of $a b$ initio total energy calculations for metals and semiconductors using a plane-wave basis set, Comput. Mater. Sci., 1996, 9, 15-50.

32 G. Kresse and J. Furthmüller, Efficient iterative schemes for $a b$ initio total-energy calculations using a plane-wave basis set, Phys. Rev. B: Condens. Matter Mater. Phys., 1996, 54, 11169.

33 J. P. Perdew, K. Burke and M. Ernzerhof, Generalized gradient approximation made simple, Phys. Rev. Lett., 1996, 77, 3865.

34 J. Heyd, G. E. Scuseria and M. Ernzerhof, Hybrid functionals based on a screened Coulomb potential, J. Chem. Phys., 2003, 118, 8207-8215.

35 A. V. Krukau, O. A. Vydrov, A. F. Izmaylov and G. E. Scuseria, Influence of the exchange screening parameter on the performance of screened hybrid functional, J. Chem. Phys, 2006, 125, 224106.

36 J. Sun, H. T. Wang, J. He and Y. J. Tian, $A b$ initio investigations of optical properties of the high-pressure phases of ZnO, Phys. Rev. B: Condens. Matter Mater. Phys., 2005, 71, 125132.

37 H. J. Monkhorst and J. D. Pack, Special points for Brillouinzone integrations, Phys. Rev. B: Solid State, 1976, 13, 188. 
38 J. Paier, M. Marsman, K. Hummer, G. Kresse, I. C. Gerber and J. G. Ángyán, Screened hybrid density functionals applied to solids, J. Chem. Phys., 2006, 124, 154709.

39 J. Heyd, G. E. Scuseria and M. Ernzerhof, Hybrid functionals based on a screened Coulomb potential, J. Chem. Phys., 2003, 118, 8207-8215.

40 C. Adamo and V. Barone, Toward reliable density functional methods without adjustable parameters: the PBE0 model, $J$. Chem. Phys., 1999, 110, 6158.

41 P. L. Silvestrelli, van der Waals interactions in DFT made easy by Wannier functions, Phys. Rev. Lett., 2008, 100, 053002.

42 P. L. Silvestrelli, van der Waals interactions in density functional theory using Wannier functions, J. Phys. Chem. A, 2009, 113, 5224-5234.

43 L. Andrinopoulos, N. D. M. Hine and A. A. Mostofi, Calculating dispersion interactions using maximally localized Wannier functions, J. Chem. Phys., 2011, 135, 154105.

44 Z. D. Li, X. J. Huang, D. X. Wu and K. M. Xiong, Large crystal growth and measurement of electro-optical coefficients of ADP, J. Cryst. Growth, 2001, 222, 524-527.

45 X. E. Ren, D. L. Xu and D. F. Xue, Crystal growth of KDP, ADP, and KADP, J. Cryst. Growth, 2008, 310, 2005-2009.

46 C. G. Van de Walle and J. Neugebauer, First-principles calculations for defects and impurities: applications to IIInitrides, J. Appl. Phys., 2004, 95, 3851-3879.

47 A. F. Kohan, G. Ceder, D. Morgan and C. G. Van de Walle, First-principles study of native point defects in ZnO, Phys. Rev. B: Condens. Matter Mater. Phys., 2000, 61, 15019.

48 J. He, R. K. Behera, M. W. Finnis, X. Li, E. C. Dickey, S. R. Phillpot and S. B. Sinnott, Prediction of hightemperature point defect formation in $\mathrm{TiO}_{2}$ from combined $a b$ initio and thermodynamic calculations, Acta Mater., 2007, 55, 4325-4337.

49 H. X. Xu, D. Lee, J. He, S. B. Sinnott, V. Gopalan, V. Dierolf and S. R. Phillpot, Stability of intrinsic defects and defect clusters in $\mathrm{LiNbO}_{3}$ from density functional theory calculations, Phys. Rev. B: Condens. Matter Mater. Phys., 2008, 78, 174103.

50 J. Zaffran and M. Caspary Toroker, Benchmarking density functional theory based methods to model $\mathrm{NiOOH}$ material properties: Hubbard and van der Waals corrections vs. hybrid functional, J. Chem. Theory Comput., 2016, 12, 3807-3812.

51 T. Le Bahers, M. Rérat and P. Sautet, Semiconductors used in photovoltaic and photocatalytic devices: assessing fundamental properties from DFT, J. Phys. Chem. C, 2014, 118, 5997-6008.

52 E. P. O'Reilly and J. Robertson, Theory of defects in vitreous silicon dioxide, Phys. Rev. B: Condens. Matter Mater. Phys., 1983, 27, 3780 .
53 Y. G. Jin and K. J. Chang, Mechanism for the enhanced diffusion of charged oxygen ions in $\mathrm{SiO}_{2}$, Phys. Rev. Lett., 2001, 86, 1793.

54 A. Ambrosetti and P. L. Silvestrelli, Adsorption of rare-gas atoms and water on graphite and graphene by van der Waals-corrected density functional theory, J. Phys. Chem. C, 2011, 115, 3695-3702.

55 P. L. Silvestrelli and A. Ambrosetti, Inclusion of screening effects in the van der Waals corrected DFT simulation of adsorption processes on metal surfaces, Phys. Rev. B: Condens. Matter Mater. Phys., 2013, 87, 075401.

56 P. L. Silvestrelli and A. Ambrosetti, van der Waals corrected DFT simulation of adsorption processes on transition-metal surfaces: Xe and graphene on Ni (111), Phys. Rev. B: Condens. Matter Mater. Phys., 2015, 91, 195405.

57 P. L. Silvestrelli, van der Waals interactions in density functional theory by combining the quantum harmonic oscillator-model with localized Wannier functions, J. Chem. Phys., 2013, 139, 054106.

58 A. Ambrosetti and P. L. Silvestrelli, van der Waals interactions in density functional theory using Wannier functions: improved $\mathrm{C}_{6}$ and $\mathrm{C}_{3}$ coefficients by a different approach, Phys. Rev. B: Condens. Matter Mater. Phys., 2012, 85, 073101.

59 S. D. Chakarova-Käck, E. Schröder, B. I. Lundqvist and D. C. Langreth, Application of van der Waals density functional to an extended system: adsorption of benzene and naphthalene on graphite, Phys. Rev. Lett., 2006, 96, 146107.

60 Y. L. Li, W. G. Schmidt and S. Sanna, Intrinsic $\mathrm{LiNbO}_{3}$ point defects from hybrid density functional calculations, Phys. Rev. B: Condens. Matter Mater. Phys., 2014, 89, 094111.

61 Y. L. Li, S. Sanna and W. G. Schmidt, Modeling intrinsic defects in $\mathrm{LiNbO}_{3}$ within the Slater-Janak transition state model, J. Chem. Phys., 2014, 140, 234113.

62 A. Yokozawa and Y. Miyamoto, First-principles calculations for charged states of hydrogen atoms in $\mathrm{SiO}_{2}$, Phys. Rev. B: Condens. Matter Mater. Phys., 1997, 55, 13783.

63 L. L. Zhu, B. A. Liu, L. S. Zhang, Q. H. Zhang, Z. P. Wang and X. Sun, Phase matching characteristics of deuterated ammonium dihydrogen phosphate crystals, Chin. opt. Lett., 2015, 13, 041601.

$64 \mathrm{~W}$. L. Smith, KDP and ADP transmission in the vacuum ultraviolet, Appl. Opt., 1977, 16, 1798.

65 C. S. Liu, Q. Zhang, N. Kioussis and S. G. Demos, Electronic structure calculations of intrinsic and extrinsic hydrogen point defects in $\mathrm{KH}_{2} \mathrm{PO}_{4}$, Phys. Rev. B: Condens. Matter Mater. Phys., 2003, 68, 224107.

66 A. Dhar and A. Mansingh, Optical properties of reduced lithium niobate single crystals, J. Appl. Phys., 1990, 68, 5804-5809. 